\title{
2006-2114: REDEVELOPING THE MECHANICS AND VIBRATION LABORATORY: A PROBLEM SOLVING APPROACH
}

\section{Mohammad Elahinia, University of Toledo}

Mohammad H. Elahinia (Ph.D., Virginia Polytechnic Institute and State University, 2004) is an assistant professor in the Department of Mechanical, Industrial and Manufacturing Engineering at the University of Toledo, where he also serves as the Co-Director for the Dynamic and Smart Systems Laboratory.

\section{Constantin Ciocanel, University of Toledo}

Constantin Ciocanel graduated with bachelor's, master's and Ph.D. degrees in mechanical engineering from the "Gh. Asachi” Technical University of lasi, Romania. He served as a lecturer in the Strength of Materials Department at the same university, where he taught undergraduate and graduate level courses on Strength of Materials, Elasticity and Finite Element Analysis. Presently, he is a Research Associate at the Dynamic and Smart Systems Laboratory at the University of Toledo (Ohio, USA). He has expertise in elasticity, piezoceramics and field responsive particulate suspensions. He is coauthor of 27 publications in international journals and conference proceedings. His current interest is in magnetorheological (MR) fluids and magneto-mechanical characterization of magnetic shape memory alloys. 


\title{
Redeveloping the Mechanics and Vibration Laboratory: A Problem Solving Approach
}

\begin{abstract}
Abstarct
This paper presents the redevelopment method and process of the laboratory experiments for the Mechanics and Vibration Laboratory, MIME3390, in the Mechanical, Industrial, and Manufacturing Engineering Department at the University of Toledo. The redevelopment objective was to transform the learning process from a subject-based learning to a problem-solving learning. Particular objective was to provide the students with more hands-on experience and to challenge them by requesting the procedure for each laboratory experiment to be designed and carried out by each group of students.

This senior level laboratory course consists of experiments in deformable solid mechanics including stress and deflection analysis, fatigue life evaluation, stability and mechanical vibration. Prerequisite courses for this laboratory are Mechanical Design I and Mechanical Vibrations. In line with the program objectives of the department, the following list of objectives has been defined for this course: "Upon successful completion of this course, the students should have: (1) become knowledgeable in the use of standard instrumentation for static and dynamic structural testing, such as strain gages, load frames, impact hammers, and spectrum analyzers; (2) reinforced material studied in previous mechanics and vibrations courses; (3) improved data analysis skills, and (4) further developed laboratory and technical writing skills."
\end{abstract}

Prior to this redevelopment, as part of the subject-based approach, a classroom lecture preceded each laboratory session. The lecture consisted of the review of the theory pertaining to each experiment to help students refresh their knowledge on the subject. Additionally the description and procedure of the laboratory experiment was covered during this lecture. Prior to each class, the lecture notes, along with the laboratory procedures, were posted on the course website. The step-by-step instructions for each experiment were provided to assist the students in setting up and conducting each experiment. Throughout the semester, eleven experiments were performed.

The students wrote individual reports on the experiments consisting of a summary of the acquired data, data analysis, and observations. However, due to the number of students and limited number of lab sessions it was difficult to provide the students with the real hands-on experience with the instrumentation and lab setup. As a result, during the lab the student mostly collected data according to the lab procedure and compiled a report that sometimes was inspired by samples of reports written by former students.

\section{Background on MIME3390}

MIME3390 (Mechanics and Vibration Laboratory) is a large-enrollment laboratory course in the Mechanical, Industrial, and Manufacturing Engineering Department at The 
University of Toledo. The main objective of the course is for the students to develop a better understanding of basic mechanics and vibration principles. Prior to the redevelopment, the laboratory was for the most part, a subject-based learning process. A classroom lecture preceded each laboratory session. The lectures covered the theory pertaining to each experiment to help students refresh their knowledge on the subject. The instruction for each experiment was also provided to assist the students in setting up and conducting each lab.

The analysis of the student feedback and the instructors' observations over the three semesters prior to the redevelopment reveals the following drawbacks of the subjectbased teaching approach, which was used to teach the course:

- The lab was structured such that students were required to complete a task rather than solving a problem. This approach, in a way, is the opposite of what an engineering graduate is required to do at the workplace, where they are "paid to formulate and solve problems that neither follow from the material presented in the book chapter nor have a single "right" answer that one can find at the end of a book" 1 .

- The individual involvement toward problem identification and formulation of the solution method was limited or nonexistent.

- Only one or two students in a group were usually involved in carrying out the experiments while the other members just made only an act of presence in the laboratory. As a result, the latter were often unable to explain the strategies used to solve the problem. The lack of individual involvement has accordingly hampered the achievement of at least one course objectives.

- The student's grading was done based on the completeness of the submitted report. A grading sheet was available for every laboratory report. The students used this to check whether in the report they addressed all the topics contributing to their grade. This approach yielded relatively standard reports for each experiment and not giving the students the opportunity to distinguish themselves through best answers to a problem within available resources and restraints. Another significant disadvantage of this approach was that many students compiled their reports using samples of reports written by former students.

- The provided standard laboratory procedure did not allow for active inter-group collaborations or discussion of the approach taken in addressing the problem. This limited the individual involvement as well as the development of teamwork skills for the students.

- Insufficient experimental set-ups or testing equipment also contributed to some extend to the above-mentioned problems.

Overall, it can be concluded that the previous methodology limited the students' initiative in structuring and writing the laboratory reports. Also, the feedback provided to the students with each graded lab report was not helping them improve their technical writing as it was focused on the correctness of the answers to the predefined questions rather than on independent interpretation and analysis of the results. In our opinion, this approach 
contributed to the erroneous evaluation of the student proficiency on writing technical reports.

\section{Problem-solving approach}

The effect of creative education methodologies on student learning have long been the focus of educational researchers. Fink et al. emphasized the need for new methods for engineering education. Through case studies, they showed the effectiveness of such methods in improving the teaching in different engineering disciplines ${ }^{2}$. The authors rightfully count the integration of major components, such as learning tools, learning activities, and learning evaluation, of the course as one of the major aspects of effective teaching. They conclude that by a proper combination of these major components one can improve the students' learning. Freuler et al. reported on their effort in the College of Engineering at the Ohio State University where they redeveloped the freshman engineering casses to a combined course with hands-on laboratory elements ${ }^{3}$. Teamwork, project management, report writing, and oral presentations were the main parts of this program.

Recently, in their research, Smith et al. focused on classroom-based pedagogy of engagement ${ }^{1}$. The authors recognized the active and collaborative learning as better ways for students to learn by being intensely involved in the educational process. These learning methods can further be implemented by encouraging the students to apply their knowledge in many situations. The article, as illustrated in Figure 1, also indicates the superiority of the problem-based learning over the subject-based learning. The authors have identified the following attributes of the first learning method: (1) Learning is student-centered (2) Learning occurs in small student groups (3) Teachers are facilitators or guides (4) Problems are the organizing focus and stimulus for learning (5) Problems are the vehicle for the development of clinical problem-solving skills (6) New information is acquired through self-directed learning. More importantly, the problemsolving approach prepares the students for formulating and solving problems they have never been exposed to before.

The Mechanical Engineering Department at Virginia Polytechnic Institute and State University has been using a problem solving approach to teach undergraduate laboratories ${ }^{4,5}$. The laboratory interweaves instruction on engineering principles with instruction on engineering communication. Using this approach, advanced topics has been successfully taught to undergraduate students ${ }^{6}$. At the Central Connecticut State University, Prusak applied the problem-solving approach in order to develop and improve important students' skills through laboratory experiments ${ }^{7}$. The students were given limited guidance to develop a projectile device. The experience was aimed at giving students the possibility of guided practice without clearly defined boundaries. The author reports positive outcomes in terms of inter-team communications and organizing.

Morgan and Jones have studied the importance and effectiveness of using computer simulations in engineering education ${ }^{8}$. According to this and similar studies, by using 
engineering software it is possible to motivate the students and to provide learning at a number of levels including cognitive and emotional. The authors further elaborated on their experience in using MATLAB for teaching a course on control systems. According to the authors' experience, the use of engineering simulation software could help the students with the process of decision-making and problem solving associated with the discipline of engineering. They also noted that the students find pleasure in the computerbased laboratory exercises. Many publications on engineering education emphasize the importance of teaching the principles. Pitts, using several examples, shows the importance of basic engineering principles in each discipline ${ }^{9}$.

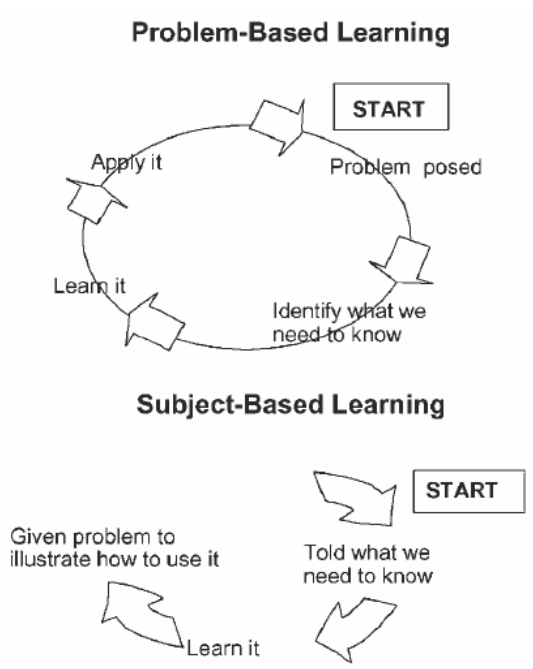

Figure 1 - Problem-solving learning contrasted with subject-based learning ${ }^{1}$

In redeveloping the Mechanics and Vibration Laboratory, several methods were considered in order to improve students' learning and to ensure achieving the course objectives. Consequently, a problem solving approach was chosen. In the redeveloped course, the number of experiments for each semester is reduced and the step-by-step lab procedures are no longer provided to the students. Instead, a practical problem is designed around each experiment. A memorandum describes each of the problems that the students must solve by performing the experiment. In other words, the redeveloped MIME3390 is a problem solving-based course to provide the students with experience in experimental investigation of mechanical engineering systems. Additionally, in the redeveloped course for some of the experimnets it is required that the students simulate the behavior of mechanical systems and compre them with experimental results.

During the term, the students work on six engineering problems that range from designing a linear displacement sensor to investigating the torsional vibration in an engine crankshaft. Each of these problems is described in a memorandum to the students from a supervisor, who defines the purpose of the problem and defines the audience for the report. Students are not given a procedure to follow for conducting the experiment. In the lab, they design the experimental procedure based on their engineering judgments and for some experiments the students make the actual test specimen. Students are introduced 
to each problem by a lecture that is followed by two sessions of lab time and an additional lecture. The lectures are intended to provide the necessary background the students will need to perform each experiment. Potential student questions related to the experimental procedure designed by the group for a particular test are being addressed either by the teaching assistants during the lab or by the instructor during the office hours between the two lab sessions. The second lecture time is for the students to clarify any ambiguities they might have about the lab. During the same session, students deliver presentations on the previous experiment. For most experiments, the students are asked to perform simulations, using MATLAB/Simulink, in order to develop a better understanding of the difference between the theoretical and experimental behavior of the system.

\section{Elements of the problem solving approach}

In redeveloping the course, the main objective was to improve the student learning. This was accomplished mainly by changing the teaching approach to problem solving. To this end, students are required to solve practical engineering problems. Each of these problems is described in a memorandum from a supervisor, who defines the purpose of the problem and defines the audience for the communication. For each laboratory problem, students attend two lectures that discuss the engineering principles of those problems and attend two laboratory sessions in which they make measurements to understand and address the problem. The redevelopment also involved interweaving the instruction on engineering principles with instruction on engineering communication. To this end, after submitting each report, each student revises the report based on the comments from the teaching assistant and the professor and resubmits the report for grading. Additionally, the student presentations are effectively incorporated into the laboratory course. Each presentation is followed by students addressing the critiques from the faculty, graduate teaching assistants, and another group of students. Incorporation of the presentations has enhanced the understanding by the students of the technical issues of the laboratory. The course consists of six laboratory problems that students work on in teams, but communicate in individual reports (and for two problems, in team presentations).

The following section present additional information about the problem-solving approach with more details on one of the lab experiments. The redeveloped experiment is compared with the previous format of the similar experiment.

\section{Experiment I: cantilever beam vibration}

In the cantilever vibrating beam experiment, the students are required to use a modal testing experimental setup, as shown in Figure 2, to identify the vibration characteristics

of a cantilever beam. 


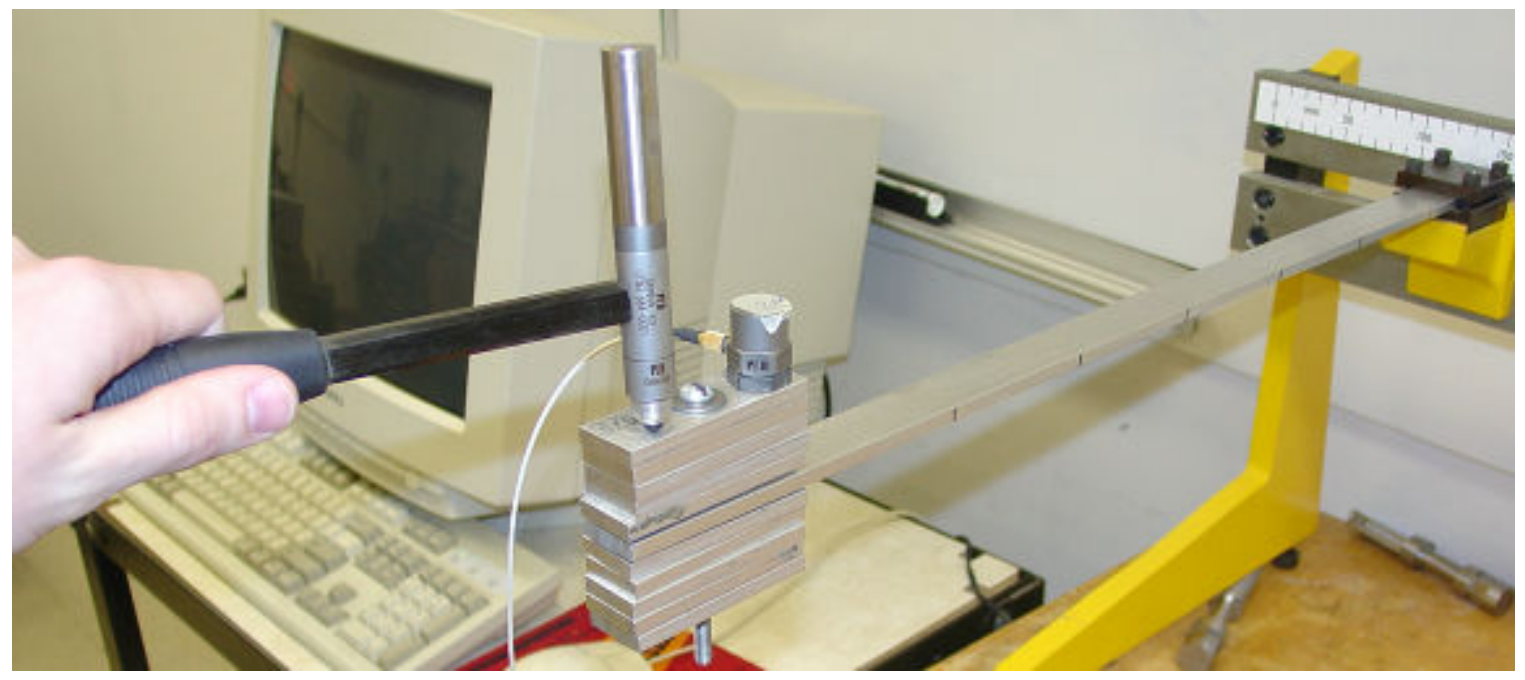

Figure 2 - Modal analysis experimental setup for the cantilever beam

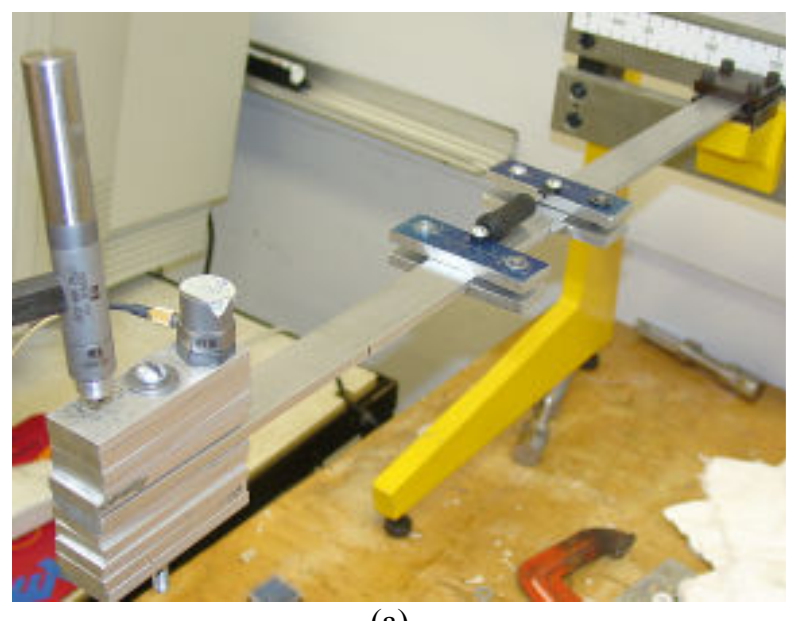

(a)

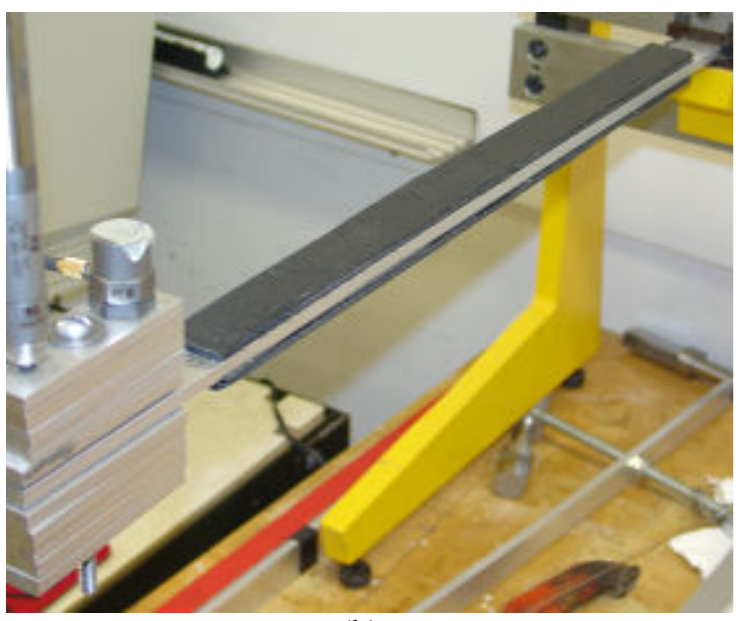

(b)

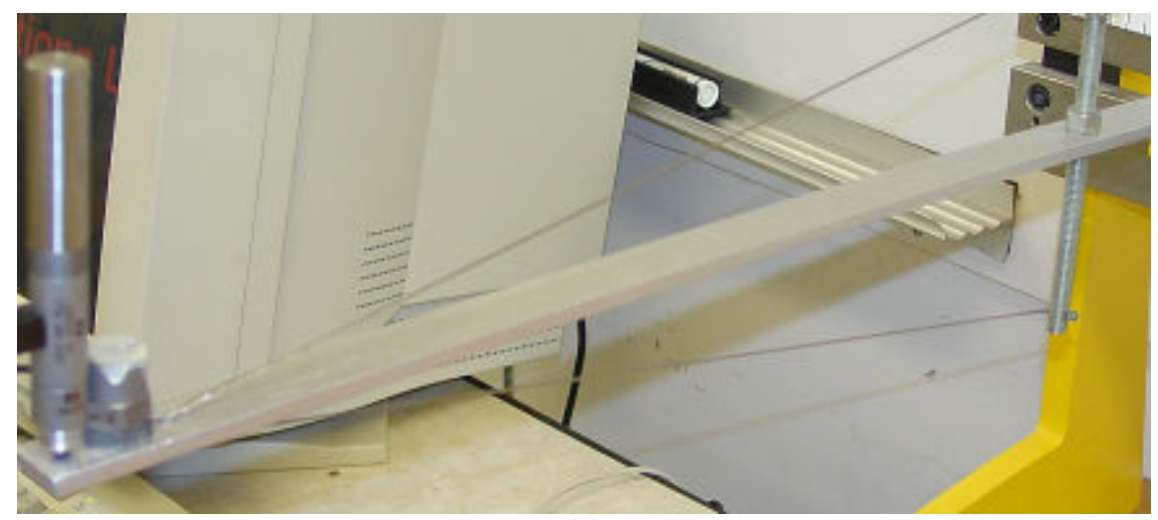

(c)

Figure 3 - Added damping solutions that was developed by the students to increase the damping of the cantilever beam which represents aircraft wing vibration behavior; viscous damper (a) damping layers (b) tension cables (c) 
Previously, a step-by-step lab procedure, as shown in appendix A, was provided for the students. This procedure highlighted the main steps of the experiment and guided the students through the data collection process in the lab. During the redevelopment process, the same experimental setup was used but students were challenged through a different scenario. A memorandum, as shown in Appendix B, from a company that works in the field of structural dynamics outlined a technical problem. The problem was to evaluate the modeling procedure that was used by the company to design aircraft wings. To this end the students were requested to experimentally test the adequacy of a linear onedegree-of-freedom lumped parameter model in describing the behavior of airplane wing by testing a cantilever beam. Furthermore, they were to test the effect of an added fuel pod, which is going to provide the extended flight range for the aircraft, on the vibration characteristics of the wing. Finally, the students were required to find two solutions for improving the damping of the wing: An immediate solution to increase the damping and reducing the adverse fluttering of the wing and a more advanced solution for next generation aircraft. Three of the solutions that were developed and tested by the students in lab are shown in Figure 3. For the more advanced damping solution, the students were asked to provide the proof-of-concept details based on the simulation results of a controllable magnetorheological damper in MATLAB/Simulink.

\section{Results}

We found the methodology developed in MIME3390 a suitable platform for improving student understanding of fundamental engineering principles. The major benefit of using this method is that the students' learning is considerably enhanced. The enhancement can be attributed to the facts that the students have to design the procedure for running the test and collecting data, they need to look at the problem from multiple perspectives and as engineers, they deal with solving practical issues related to the problem. The other factor that plays an important role in improving the learning is that the students have to present their results; these presentations are critiqued by faculty, graduate teaching assistants, and peer students.

Two sets of student evaluations were administrated during the semester, one at the middle of the semester after the completion of three experiments related to the mechanical part of the lab, and one at the end of the semester. The mid semester evaluation was used to identify ongoing issues, specific to the new approach, and to address them accordingly. The end of semester evaluation, more relevant for the outcome of the redevelopment, was compared with the students' feedback from the past four semesters (see Figure 4). The questionnaire answered by the students (Appendix C Survey 1/3) addresses the level of achievement of the outcomes of the course. The summary of the results (see Figure 4) indicates that overall the redevelopment was successful. It is worth noting that the curved beam memo/experiment included a small research component. Specifically, the students were required to use two bibliographical sources for the normal stress calculation. One of these two texts was printed in a foreign language. This task proved to be quite difficult and it clearly negatively impacted the 
overall perception relative to the theoretical understanding of normal stress calculation in curved beams. The answer average on question 3 was expected to be five as no experiment or calculations involving stresses in pressure vessels was involved in the redeveloped lab course. It is believed that some students answered this question in relation to their general knowledge on pressure vessels stress calculation. The ability to write a lab report was overestimated in previous semesters as for every experiment the students had to compile an almost standard report. In the problem solving approach, however, the students had to assess independently the information relevant for the beneficiary of the report, to structure the report such that it would be easy to read and to make sure the report was technically sound.
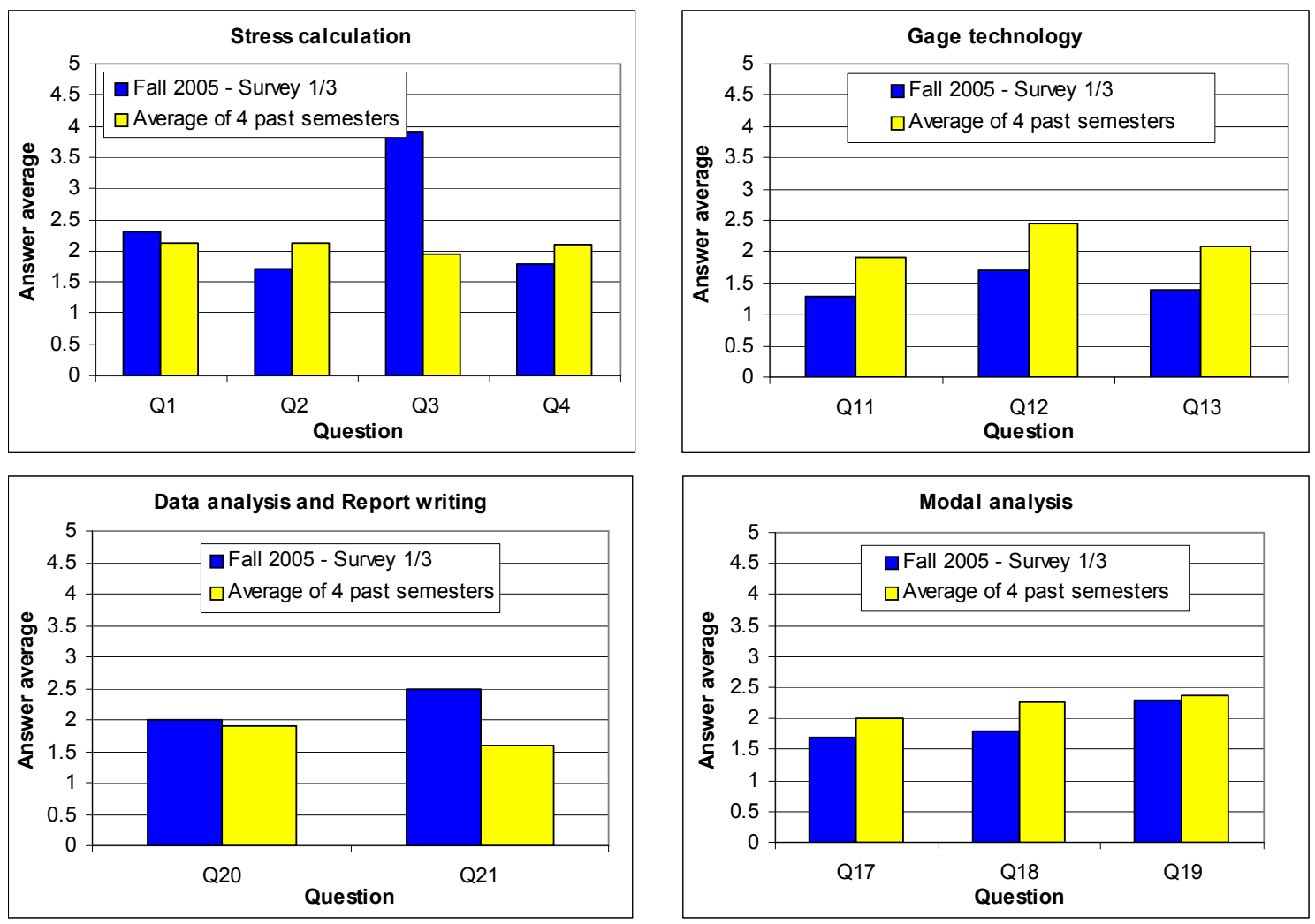

Figure 4 - Comparison between the level of achievement of the course's outcome at the end of the current semester and the average of the same from the past four semesters

In this approach, the students were required to submit a first draft of the report for each experiment. This draft was evaluated by the instructor or the teaching assistants then was it was returned to the students for revisions. After addressing the suggested revisions, the second and final report was submitted by the students. The contribution to the final grade was $40 \%$ for the first draft and $60 \%$ for the final report. Communicating the students their mistakes helped them improve their technical writing skills and understand what information needs to be included in a technical report. Since this type of grading was not 
previously used in the course, it is believed that the outcome covered by question 21 was highly overrated as shown in Figure 4.
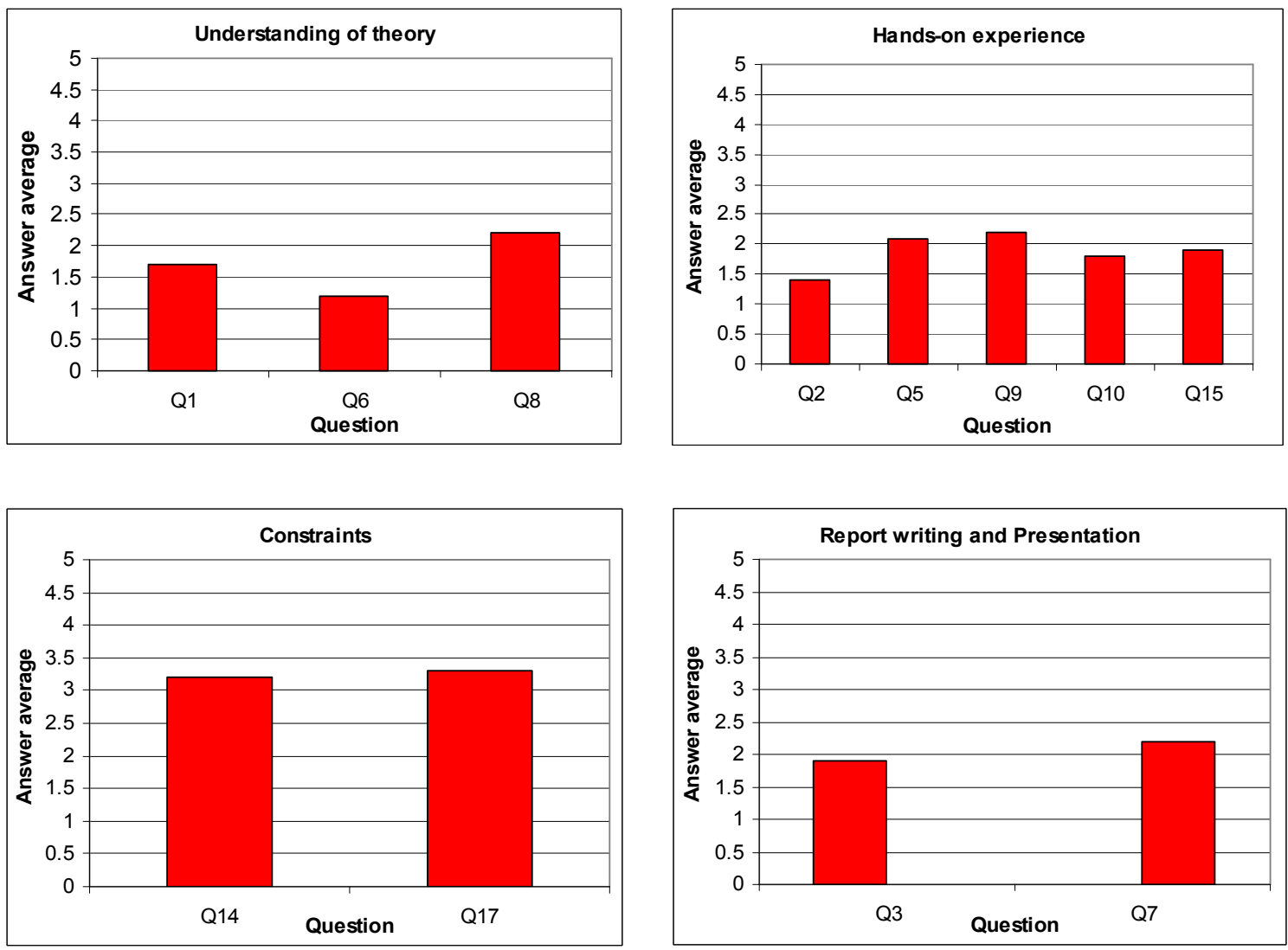

Figure 5 - Results of the student evaluation administrated at the end of the semester during which the change in teaching approach occurred. The questions are provided in Appendix C - Survey 2/3

In addition to the questions related to the outcomes of the the course, a secnd set of questions were designed to evalaute the studets' learning as shown in Appendix C Survey $2 / 3$. The focus of the second questionare is on four categories: 1) understanding of the theory and its limitations, 2) efficiency of the hands-on approach, 3) constraints and limitations students were faced with in the course, and 4) presentation and technical writing expertise. The number of questions in each category is different: three questions for the $1^{\text {st }}$ category, five questions for the $2^{\text {nd }}$, and two questio for the $3^{\text {rd }}$ and $4^{\text {th }}$ category. The results are summarized in Figure 5. Similar to previous survey, the scale was from 1 to 5 , with 1 equivalent to strongly agree and 5 strongly disagree.

A high average, equivalent to strong student disagreement on the proposed approach, has been recorded for the constraints category (see Figure 5). Most students considered the amount of time required to complete each laboratory experiment and the corresponding laboratory report was much more than expected for a two credit hour course. However, while the time component had a negative impact on the students' feedback, the 
educational benefits of the approach was recognized by the students. This is indicated by the relatively low average values (meaning high level of approval) on theory, hands-on, and report writing and presentation indicators. The hands-on experience was very appreciated and many students agreed that it helped them better understand the theoretical concepts. Allocating more time for the completion of the experimental setups and data acquisition would probably improve the ratings of this category and would affect positively the constraints category as well. Allowing the students to work independently on the experimental setups was also beneficial for the better understanding of the theory. During the lecture time, as well as during the preparation of the experiments, questions were asked on the impact of the theoretical assumptions on the results, on why certain approximations have been made in the theoretical development, on what is the acceptable level of error introduced by such simplifications, etc. Finally, the report writing and presentation category received higher marks in this pool compared with the ones reported in Figure 4. This is believed to be due to the more focused questions addressing these issues.

In conclusion, we believe that the redevelopment had an overall beneficial impact on the students' learning and we are going to closely monitor the course for the next two semesters. 


\section{References}

[1] Smith, K., Sheppard, S., Johnson, D., and Johnson, R., "Pedagogies of Engagement: Classroom-Based Practices," Journal of Engineering Education, American Society of Engineering Education, January 2005, 94(1):87-101.

[2] Fink, L., Ambrose, S., and Wheeler, D., "Becoming a Professional Engineering Educator: A New Role for a New Era," Journal of Engineering Education, American Society of Engineering Education, January 2005, 94(1):185-194.

[3] Freuler, R., Fentiman, A., Demel, J., Gustafson, R., and Merrill, J. "Developing and Implementing Hands-on Laboratory Exercises and Design Projects for First Year Engineering Students," 2001 ASEE Annual Conference (Albuquerque: ASEE, June 2001).

[4] Alley, Michael, and Harry Robertshaw, "Interweaving Engineering and Writing: A Model Large-Laboratory Course at Virginia Tech," 2001 ASEE Annual Conference (Albuquerque: ASEE, June 2001).

[5] Alley, Michael, and Harry Robertshaw, "An Effective Incorporation of Student Presentations Into a Large Laboratory Course," 2001 ASEE Annual Conference (Albuquerque: ASEE, June 2001).

[6] Elahinia, M., "Teaching Smart Materials to Engineering Undergraduate Students: A Problem Solving Approach," 2004 ASEE Annual Conference (Salt Lake City: ASEE, June 2004).

[7] Prusak, Z., "Development of Engineering Problem Solving Skills Through Laboratory Experimentation," 2001 ASEE Annual Conference (Albuquerque: ASEE, June 2001).

[8] Morgan, R., and Jones, K., "The Use of Simulation Software to Enhance Student Understanding," IEE International Symposium on Engineering Education: Innovations in Teaching, Learning and Assessment (Ref. No. 2001/046), 2001, 33/1$33 / 6$.

[9] Pitts, G. "How do we teach students to relate to real applications," IEE Colloquium on Engineering Education in the Twenty-First Century (Digest No: 1996/105), May $1996,5 / 1-5 / 5$. 


\section{Appendix A: Lab procedure for cantilever beam experiment prior to redevelopment}

The primary focus of the lab is to familiarize each student with the spectral analyzer and its use to determine vibration characteristics. This equipment will be used in several of the remaining labs this semester. In this experiment, each person will perform an impact hammer test on the cantilever beam setup. The detail steps to be followed are given next.

Use a balance to measure the masses of the plates, bolt, nut and washers at the end of the beam. Place all pieces on the balance plate and take one measurement.

Measure the length, $\mathrm{L}_{\mathrm{o}}$, width, thickness and mass of the beam.

Measure the mass of the accelerometer.

Clamp the beam in the fixture and measure the distance $\mathrm{L}_{\mathrm{k}}$ between the fixed end and the point where the weight is applied. Also measure the distance L between the fixed end and the free end of the beam. If there is any question about the measurements, both $\mathrm{L}_{\mathrm{k}}$ and $L$ are shown in Figure 1.

Each student should then perform five impact hammer tests on the beam. An impact hammer test consists of softly striking the tip weight near the bolt and waiting while the system computes and displays the frequency response function. Make sure that the hammer only strikes the weight once. Sometimes the beam will rebound and strike the hammer, resulting in a "double hit" which corrupts the data. If the beam is hit too hard an overload condition will result and the entire set of five impacts will have to be repeated.

The system is programmed to take the average of five frequency response functions so attempt to hit the beam in the same location and with the same force for all of the tests. Your lab instructor will know whether the data is acceptable.

Capture the image on the screen by pushing the Print Screen button on the computer keyboard and then pasting the image into a Word document

Once an acceptable set of data has been obtained, export the measured time history and frequency response to a text file (your TA will help you with exporting the file). Use your first initial and your complete last name as the name of the text file (e.g. John Smith would be called jsmith.txt).

Load the text file into Excel and save the data as an Excel spreadsheet with the same file name (e.g. jsmith.xls).

Mail the spreadsheet and Word document containing the screen image to each member of the group.

Data and Observations

Include the following items along with explanations in your report.

A table of all measured masses.

A table of all measured dimensions. 
Pasted image of the CRT display.

Using the Excel data from the experiment, plot the time history of the acceleration at the tip of the beam

Using the Excel file from the experiment, plot the frequency response. A description of the records in the file is provided in the document titled Medallion Universal File Format on course lab web page.

List any observations you made during the experiment.

Results and Analysis

Include the following results in this section. Each calculation, table or graph should be preceded by a verbal description of what is being calculated or being displayed and how the values are determined. Any equations used should be given and the terms used in the equations should be defined. The purpose should be given for all equations and all terms should be defined. Make sure that you clearly label all points on the graphs and use proper units for the axes.

Using the plot of the time history of the acceleration, determine the natural frequency of the system. This can be done by counting the number of oscillations in a given period of time.

Using the frequency response plot, determine the natural frequency of the system. The natural frequencies are the abscissa values where spikes occur.

Determine the stiffness of the beam in $\mathrm{lbf} /$ in from experimental data using equation (3). To do this use the natural frequency obtained from the frequency response plot in a previous step and use the equivalent mass of the beam as calculated using equation (2). For the equivalent mass in equation (2), the dimensions and mass of the beam that were measured can be used to estimate the mass of the beam per unit length and then the mass per unit length times $L_{k}$ will give the mass of the beam that is vibrating, $m_{\text {beam }}$. Also the mass of the beam per unit length times the difference between $L$ and $L_{k}$ will give the extra mass that is to be included as part of $\mathrm{m}_{\mathrm{tip}}$. The additional mass at the tip is the mass of the plates, bolt, nut, washers and accelerometer that were measured directly with the balance. 


\section{Appendix B - Memorandum for cantilever beam experiment after redevelopment}

Structural Analysis Inc.

1324 N. Main St.

Blacksburg, VA 24060

October 202005

To: $\quad$ Dr. Nobel Prizewinner

From: $\quad$ Dr. Fernando D. Goncalves, Noise and Vibration Division FDG

Subject: $\quad$ Request for wing design for the autonomous reconnaissance airplane RQ2

Problem Statement

Structural Analysis Inc. is designing the new Unmanned Aerial Vehicle (UAV) currently code-named as RQ-2. This UAV will be an upgrade from the successful RQ1 Predator MAEA. According to the Air Force, the Predator is a "Joint Forces Air Commanderowned theater asset for reconnaissance, surveillance, and target acquisition in support of the Joint Force commander." Because the Predator is unmanned, it is suitable for deployment in "moderate risk areas", unsecured air space, "open ocean environments, and biological or chemical contaminated environments." In addition, as the October 2001 attacks in Afghanistan have illustrated, the Predator can now perform a search and destroy mission with no apparent risk to US military personnel.

RQ1 has an endurance of 40 hours. In order to extend this operation range to 80 hours, due to the change of the mission, two fuel pods will be mounted at each wing tip as illustrated in Figure . Each pod has a mass of $1 \mathrm{~kg}$ when it is empty and $100 \mathrm{~kg}$ when it is full. Adding these pods can potentially cause excessive vibration in the wings. This increase level of vibration and noise can affect the accuracy and sensitivity of the surveillance equipments. Structural Analysis Inc. has adapted a new noise and vibration standard for the surveillance equipments. According to this standard in order to maintain the accuracy of these equipments as well as the structural integrity of the UAV, the damping coefficient of the wings should remain in the range of $0.7<\zeta<0.9$.

\section{Background}

It is well known that the natural frequency of vibration of the wings changes as the airplane consumes the fuel in the wing pods. Our main objective is that the wing structure maintains the desired vibration characteristics over the entire flight time. This memo requests that your engineering team tests and reports the vibration flutter characteristics a wing model made of similar material. Our company is also working on the initial stage of the design of the next generation of similar UAV. To maintain the continuity of the design process we request that your team provide two solutions: In addition to (1) a damping method that can be immediately implemented on RQ-2, we would like (2) a 
proof of concept simulation for the next generation. To address the first requirement please ask your team members to modify the existing lab setup and to use experimental data for showing the improved damping characteristics. To this end, you need to add components to the existing setup in order to achieve higher damping. The solution must be implemented in a two-week period. An example of such a solution is to add damping material to the top and lower surface of the wing. For the proof of concept simulation, please use the Simulink model available on our webpage. You need to modify this model by designing a logic that would change the damping coefficient. This variable damping element will represent the effect of an advanced Magnetorhelogical (MR) damper. Details on this type of dampers are also available on our company's website. We will use the logic provided by your team to control the MR dampers that we intend to incorporate in the next generation UAVs. The following section includes some background information related to the problem.

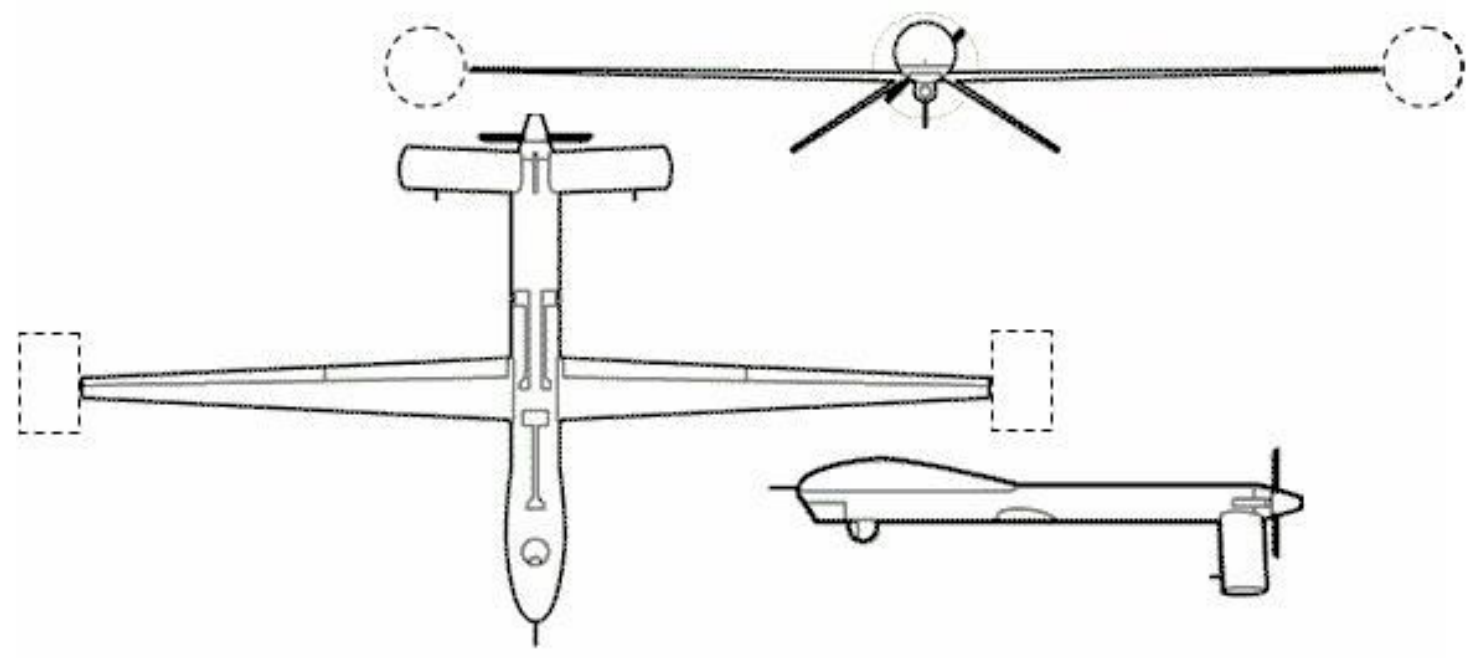

Figure 1 Unmanned Aerial Vehicle (UAV) RQ-1. To increase the endurance, one the proposed modifications for the redesigned model R-Q2, as shown by the dotted lines, is the fuel pods at the tip of the wings

In a UAV, the wing must be capable of supporting the weight of the vehicle as well as the additional lift produced during turning flight. The resulting wing structure can be viewed as a blade or spring extending from the fuselage. If we "tap" the spring with a hammer, it will vibrate at a frequency which relates to the stiffness of the spring. A stiff spring will vibrate at a higher frequency than a more limber spring. This frequency is known as the "natural frequency" of the spring. "Flutter" is the term used for synchronized vibration when it takes place in a flexible structure moving through a fluid medium--for instance, an airplane in flight. It occurs when two regular, rhythmic motions coincide in such a way that one feeds the other, drawing additional energy from the surrounding flow. 


\section{Appendix C, Student evaluation surveys}

Survey $1 / 3$

Answer each question as it relates to the course or was demonstrated in the course. Use the following scale. 1 = excellent level, 2 = high level, 3 = adequate level, $4=$ below the level expected, $5=$ little or no knowledge or skill or topic or problem area was not covered in this course.

1. How well can you calculate stresses in curved beams?

2. How well can you calculate fatigue life and stress in rotating bending fatigue?

3 . How well can you calculate stresses in thin wall pressure vessels?

4. How well can you calculate principal stresses from strain gage rosette data?

5 . How well can you calculate stresses produced by torsion and bending?

6 . How well can you calculate free undamped and damped vibration motion of single degree of freedom linear and rotational systems?

7. How well can you calculate free undamped vibration of multi-degree of freedom linear and rotational concentrated mass systems including eigenvalue and eigenvector solutions?

8. The solution to some of the problems in this course involves using calculus. What is the level of your ability to solve the problems involving calculus?

9. The solution to some of the problems in this course involves using differential equations. What is the level of your ability to solve the problems involving differential equations?

10. This course involves solving problems using basic physics. What is your level of knowledge in solving problems using basic physics?

11. How well do you know the method to mount strain gages?

12. What is your ability to wire strain gages into a Wheatstone bridge circuit?

13. How well do you understand temperature compensation of strain gage circuits?

14. What is the level of your ability to maximize desirable strain output or minimize undesirable strain output using gage orientation and gage position in the bridge circuit?

15. What is the level of your ability to use an Instron tension/compression testing machine?

16. What is the level of your ability to use a rotating bending fatigue machine?

17. What is your ability level to use horizontal and vertical shakers to determine natural frequencies and mode shapes?

18. What is the level of your ability to connect accelerometers to amplifiers and spectral analyzers?

19. What is the level of your ability to use the output from single and multi-channel input to determine equivalent mass, equivalent spring constant and equivalent-damping ratio for a single degree of freedom system?

20. How well can you analyze experimental data?

21. How well can you write a laboratory report? 
Survey $2 / 3$

Please use the scale of 1 (strongly agree) 2 (agree) 3 (not sure) 4 (don't agree) 5 (strongly disagree) to assess the following statements.

The lab helped me to understand the theory better.

The hands-on approach of this lab helped me learn more.

I improved my technical writing skills through revising the reports based on the instructor's comments.

I used the recommended textbooks to prepare better reports and presentations.

I contributed in designing the procedure for the experiments.

In writing the reports, I compared theoretical and experimental results and I was asked to explain the difference.

The class helped me improve my presentation skills.

I learned to consider limitations and underlying assumptions of a theory before applying it to solve a problem.

After solving engineering problems in this course, I feel I am more prepared as an engineer.

Instead of receiving a step-by-step lab procedure, I think it is more beneficial for the students to receive a problem to solve by performing the experiment.

The memos stated clearly the problems to be investigated.

The information provided in the lectures, memorandums, and supplemental materials on the web were enough to understand the problem and to conduct the experiment.

The laboratory testing procedures were easy to develop based on the information provided in memos and lectures.

There was enough time allocated for the completion of the lab experiments.

After completing the course, I feel confident to set up and perform independently (or with minimal assistance) a basic stress or vibration related experiment.

I learned to simulate the behavior of a 1-DOF and multi-DOF system through

MATLAB/Simulink.

The amount of time required to complete the lab reports was acceptable.

I will definitively recommend this class to all my engineering majoring friends.

Survey $3 / 3$

Please briefly describe your experience with this lab. 